\title{
XVIII. Ueber ein Instrument zum Schleifen von genau orientirten Platten und Prismen künstlicher Krystalle.
}

\author{
Von
}

\author{
A. E. Tutton in London*).
}

(Mit 3 Textfguren.)

Bei der optischen Untersuchung künstlich hergestellter Krystalle, die meist einen viel geringeren Grad von Härte besitzen, als die in der Natur vorkommenden Mineralien, ist die erste und schwierigste Aufgabe die, die nöthigen Platten und Prismen herzustellen. Es ist, wenn die Untersuchungen genau ausfallen sollen, von fundamentaler Wichtigkeit, dass die Platten wirklich parallel zu der gewunschten Ebene, oder wirklich senkrecht zu der gewtunschten Richtung im Krystall sind, ferner dass sie ebene und genau parallele Oberflächen besitzen. Bei Prismen mússen zwei ebene Flächen vorbanden sein, welche einen Winkel von nicht mehr als $70^{\circ} \mathrm{mit}$ einander bilden; diese Ebenen mussen sich in einer Linie schneiden, welche einer bestimmten hichtung im Krystalle genau parallel ist, und ausserdem sollen sie meist zu einer gegebenen Krystallfäche symmetrisch liegen, oder es soll die eine zu' einer Krystallfäche parallel sein. Es ist einleuchtend, dass die Genauigkeit der Bestimmungen optischer Constanten abhängt von dem Grade der Präcision, mit dem diese Bedingungen erfüllt sind.

In der Mehrzahl der Fälle zeigen die künstlichen Krystalle die zu den Prismen und Platten nöthigen Flächen entweder gar nicht oder nur untergeordnet ausgebildet, und in diesem Falle muss das verhältnissmässig weiche und zerbrechliche Material geschliffen werden. Nur selten sind künstliche Krystalle hart und widerstandsfähig genug, dass man sie zunächst mit einer feinen Laubsäge oder einem Drahte schneiden kann, welch

*) Aus dem 185. Bande der Phil. Transactions Roy. Soc. London vom Vref. mitgetheilt. '

Groth, Zeitschrift f. Krygtallogr. XXIV. 
letzterer mit Oel oder einer Lösung der betreffenden Substanz befeuchtet sein muss. Die Krystalle müssen meist sehr vorsichtig behandelt werden, da ihre Weichheit und Sprödigkeit, sowie die oft auftretende Spaltbarkeit leicht ein Zerbrechen und Zersplittern verursachen. Ausserdem eignen sich kleinere Krystalle immer besser zu cien Untersuchungen als die grösseren, da letztere viel häufiger gestreift, verzerrt oder mit gekrümmten Flächen versehen sind als erstere, und die Herstellung von Schliffen kleiner Individuen kann selbstredend nur durch Schleifen ausgefuhrt werden.

Die erste Oberfläche einer Platte wird meist in der Weise bergestellt, dass man den Krystall fest zwischen Zeigefinger und Daumen fasst und ihn vorsichtig uber der Oberfläche einer fein angeschliffenen und schwach convexen Glasplatte hin und her bewegt; dieselbe ist mit Oel oder einer Flussigkeit zu befeuchten, welche die Substanz schwer löst; man muss beim Schleifen eine Bewegung des Handgelenkes vermeiden, da dadurch die geschliffene Fläche mehr oder weniger convex wird. Wenn, was selten vorkommt, der Krystall ziemlich bart und nicht spröde ist, so kann vielleicht ein Halter aus einem gespaltenen Kork improvisirt werden, aber in den meisten Fällen muss man seine Zuflucht zum Schleifen mit Daumen und Zeigefinger nehmen. Hat man so eine Fläche angeschliffen, so wird sie auf einem Stuck Seide polirt und probirt, ob sie eben ist und approximativ die richtige Lage hat, indem man den Krystall auf's Goniometer bringt, zusieht, wie die Fläche reflectirt und mindestens zwei Winkel misst, welche sie mit am Krystall ausgebildeten Flächen bildet. Ist das Resultat nicht befriedigend, so muss von neuem geschliffen und probirt werden, bis man seinen Zweck erreicht hat. Es muss sodann auf ăhnliche Art eine zweite Fläche parallel zur ersten geschliffen werden, bis eine hinreichend, aber nicht zu dünne Platte erbalten ist, die, wenn ein zweiaxiger Krystall vorliegt, im Axenwinkelapparate die inneren Ringe der Interferenzfigur sehr klein und die Hyperbeläste, deren Abstand gemessen werden soll, am deutlichsten zeigt. Bevor man die zweite Fläche schleift, pllegı man den Krystall auf einer Glasplatte zu montiren, indem man die erste Fläche mit Canadabalsam auf derselben festkittet; man kann dann die Platte während des Schleifens besser halten, die Gefabr des Zerbrechens ist vermindert und, wenn der Krystall slark doppeltbrechend ist, die Platte also sehr dünn werden muss, erzielt man leicht annähernde Parallelität beider Flächen. Wenn die Krystalle nicht sehr klein sind, so kann man die zweite Fläche vortheilhaft unter Anwendung des zu diesem $Z$ wecke von Fuess in Berlin construirten kleinen Apparates besser parallel schleifen. Der Krystall wird mit der zuerst angeschliffenen Fläche auf das eine Ende eines geschlossenen weissen Metallcylinders aufgekittet, der etwa $5 \frac{1}{2} \mathrm{~cm}$ lang ist und $2 \frac{1}{2} \mathrm{~cm}$ Durchmesser hat, und dessen Enden eben und soviel als möglich senkrecht zur Axe sind. Der Cylinder gleitet vertical mit dem Krystalle unten in einer äusseren 
Messingröhre, von deren unterem Ende horizontal drei Arme ausgehen, die mit feingeschnittenen Schrauben versehen sind. Diese letzteren werden mit Hülfe eines getheilten Keiles so justirt, dass die Enden der Arme in derselben Höhe uber der Schleifplatte sich befinden, wenn der Cylinder senkrecht zu derselben steht. Wenn man den Apparat unter Anwendung eines leichten Druckes auf den Cylinder über die angefeuchtete Schleifplatte hin und her bewegl, so wird an den Krystall eine zweite zu der ersten parallele Fläche angeschliffen. Bei kleinen Krystallen ist diese Methode, die zweite Flăche zu schleifen, indessen nicht bequem.

Das Anschleifen der ersten Fläche eines Prismas von einem kleinen künstlichen Krystalle wird meist wie bei Platten mit der Hand ausgefuhrt; ebenso wird die zweite Fläche, die natürlich viel schwieriger in der gewünschten Richtung zu erhalten ist, gewöhnlich mit der Hand geschliffen.

Es ist klar, dass nach diesen Methoden im besten Falle Platten und Prismen von annähernd ebener Oberfläche erhalten werden können, die nur approximativ orientirt sind, denn es leuchtet obne weiteres ein, wie schwierig es ist, einen kleinen, vielleicht nur $2 \mathrm{~mm}$ langen Krystall während des Schleifens so zu halten, dass eine bestimmte Ebene, deren Lage nach den am Krystall entwickelten Flächen geschätzt werden muss, der Schleifplatte parallel ist. Ausserdem ist es selbst nach langer Uebung für gewöhnlich unmöglich, eine wirklich ebene Oberfläche zu schleifen. Der Gebrauch einer etwas convexen Schleifplatte hilft nur wenig, um die durch eine unwillkürliche Bewegung des Handgelenkes verursachte Krümmung der angeschliffenen Flüche zu eliminiren. Auch ist es eine unangenehme, aber oft gemachte Erfahrung, dass man, wenn nach vieler Mühe eine glatte, anscheinend ebene Fläche ohne Zerbrechen des Krystalles geschliffen ist, bei der goniometrischen Untersuchung Abweichungen bis zu $5^{0}$ von der gewünschten Richtung findet. Auch kommt es vor, dass durch Zerbrechen der Krystalle während des Schleifens oft viele Stunden verloren gehen. Es geht daraus hervor, dass die Herstellung einer grösseren Zahl von Platten und Prismen zum Zwecke einer ausgedehnten Untersuchung nach der gebräuchlichen Methode ungemein viel Zeit und Mühe kostet und die Geduld des Experimentators auf eine harte Probe stellt, während doch die Resultate als nur approximative bezeichnet werden mussen.

Das im Folgenden zu beschreibende Instrument ist das Resultat eines Versuches, diese ermudenden und nur approximative Resullate gebenden Melhoden durch eine Präcisionsmethode zu ersetzen, welche die ermüdende Handarbeit eliminirt und zu gleicher Zeit Garantie bietet, dass man völlig ebene und genau orientirte Flächen erhält. Der Versuch ist erfolgreich ausgefallen und es ist möglich, mittelst des Instrumentes Flächen von der besten Beschaffenheit zu schleifen, die bis auf $10^{\prime}$ mit der gewünschten Richtung zusammenfallen, ein möglicher Fehler, der auf die Werthe der optischen 
Constanten keinen messbaren Einfluss ausüben würde. Ueberdies kann dieses Resultat in einem kleinen Bruchtheile der bisher erforderlichen Zeit erzielt werden und die Gefahr, den Krystall zu zerbrechen, ist sehr verringert. Auch ist eine Vorrichtung angebracht, um mit demselben Grade von Genauigkeit eine zweite der ersten parallele Fläche zu schleifen. Für Fälle, in denen es wegen schwacher Doppelbrechung wünschenswerth ist, ziemlich dicke Platten zur Verfugung zu haben, damit die Ringe der Interferenzfigur recht klein werden, können am gleichen Krystalle zwei Paare paralleler Flächen angeschliffen. werden, die resp. zur ersten und zweiten Bisectrix senkrecht sind. Auch ist es leicht und kann bei zweiaxigen Krystallen, wie gross die Doppelbrechung auch sein möge, immer ausgeführt werden, an demselben Individuum ein Paar Prismen anzuschleifen, welche es gestatten, alle drei Brechungsexponenten an demselben Krystall zu messen. Ja, wenn Krystalle von schwacher Doppelbrechung mit drei oder mehr Millimeter Durchmesser vorliegen, so kann man an ein und demselben Individuum zwei Platten und zwei Prismen schleifen, die es gestatten, die sämmtlichen optischen Constanten zu bestimmen. Ausserdem haben die mittelst des Instrumentes erzeugten Platten und Prismen den grossen Vorzug, dass sie so glatt polirt sind, dass man sie direct ohne Anwendung von Deckgläschen benutzen kann, und die erhaltenen Resultate sind nicht mehr approximativ, sondern absolut genau.

\section{Construction des Instrumentes.}

Eine allgemeine Ansicht des Instrumentes und der zugehörigen Nebenapparate giebt nebenstebende Fig. 1.

Dasselbe besteht hauptsächlich aus den folgenden Theilen :

1) Einem drehbaren horizontalen Theilkreise mit festem Nonius.

2) Einer aufgehängten senkrechten Axe, die mit dem Theilkreise rotirt und auf und ab bewegt werden kann; am unteren Ende trägt sie den Krystall und Justirvorrichtungen.

3) Einen drehbaren Schleiftisch, dessen Oberfläche dem Theilkreise parallel und zur Axe senkrecht ist.

4) Einen horizontalen Collimator und ein Fernrohr, um den Krystall goniometrisch zu beobachten.

5) Eine Vorrichtung, um den Druck, mit dem der Krystall auf den Schleiftisch gepresst wird, zu vergrössern, zu verringern oder ganz aufzuheben.

Auf einer kreisförmigen schweren Metallplatte sind drei Messingsäulen montirt, die eine starke metallene dreieckige Verbindungsplatte mit etwas concaven Ecken tragen, in dem in Fig. 2 gegebenen Durchschnitte mit $a$ 
bezeichnet. In der Mitte dieser Platte, mit ihr aus demselben Gusse, befindet sich der äussere feste Conus $b$, in dem die verschiedenen drehbaren Axen sich bewegen. Der silberne Nonius befindet sich an einem kurzen Arme, der zwischen zwei Armen jener Platte befestigt ist. In dem äusseren

Fig. 1.

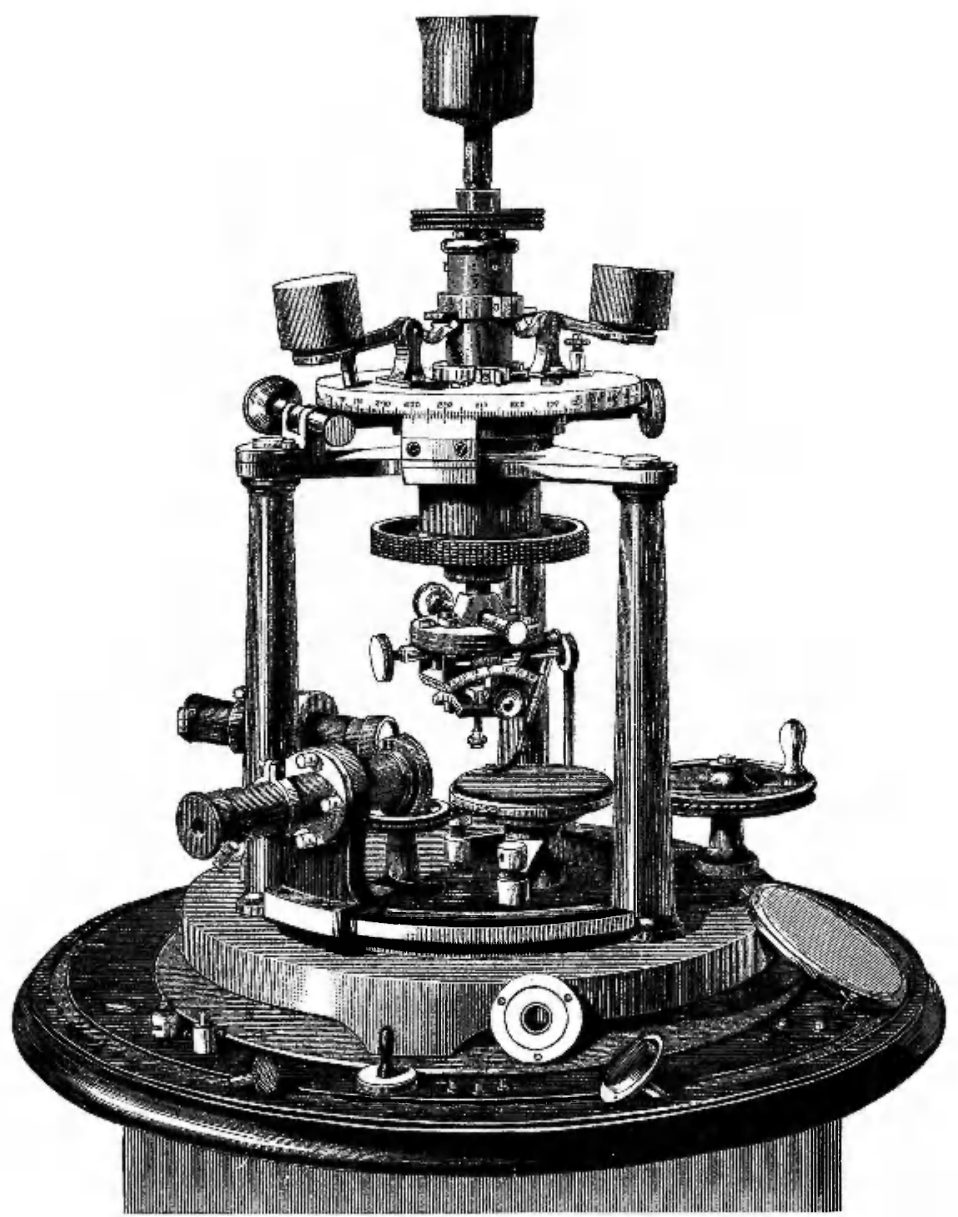

festen Conus $b$ befindet sich ein zweiter $c$, der mit Hülfe einer grossen gèrillten Ebonitplatte $d$ gedreht werden kann, welche direct unterhalb der Endigung des äusseren Conus fest mit jenem verbunden ist. Oben ist diese bewegliche Axe an dem Theilkreise $c$ festgeschraubt, so dass beim Drehen des Ebonittisches der Theilkreis sich dreht.

Auf dem Theilkreise befindet sich ein dicker Silberring, auf dem die Theilung angebracht ist; direct kann man halbe Grade, mit dem Nonius 
Minuten ablesen. Direct unterhalb des Theilkreises ist der Conus $c$ lose von einem Ringe $f$ umgeben, der gegebenenfalls mit einer Klemmschraube an demselben befestigt werden kann. Diese Klemmschraube bewegt sich in einem an dem Ringe angebrachten Arme; auf der anderen Seite des Ringes

Fig. 2.

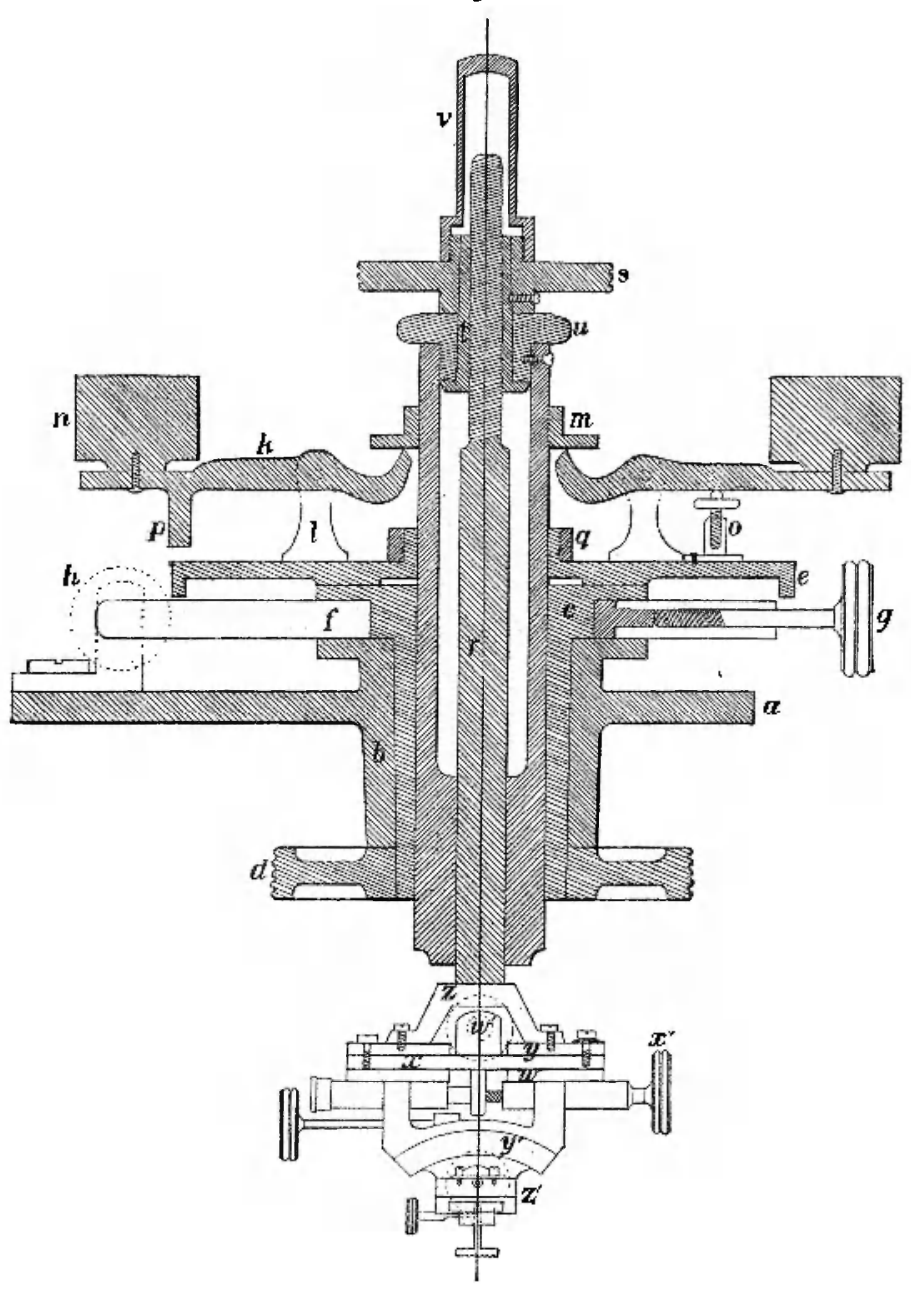

zweigt sich ein zweiter Arm ab, auf den eine Mikrometerschraube stösst; diese letztere ist ähnlich eingerichtet wie an den Fuess'schen Goniometern und ermöglicht eine präcise Einstellung des Theilkreises.

Der Winkel der conischen Bohrung im äusseren Conus $b$ ist nur klein und die Bohrung in $c$ ist genau cylindrisch. In dieser cylindrischen Röhre bewegt sich eine Axe $i$ aus Bronze, deren unabhängige Drehung verhindert 
wird durch eine der Länge nach verlaufende Kerbung, welcher ein Metallvorsprung auf der inneren Oberfläche von $c$ entspricht. Diese Axe bewegt sich also mit dem Theilkreise, kann aber frei auf und ab bewegt werden. Sie wird durch ein Paar Hebel $k$ in passender Höhe gehalten; diese sind an den Enden ziemlich schwer belastet; ihre Drehpunkte sind auf dem Theilkreise befestigt und ihre kürzeren, gebogenen Arme drücken nach aufwärts gegen einen gespaltenen Ring $m$, der an der Axe durch eine mit einem Schlussel anzuziehende Schraube befestigt werden kann. Die Gegengewichte $n$ sind so justirt, dass, wenn die Hebelarme nahezu horizontal stehen, das ganze Gewicht von der Axe $i$ mit allem, was sie trägt, im Gleichgewicht gehalten wird und die geringste Berührung der Hebelarme genügt, um die Axe auf und ab zu bewegen. Die Axe $i$ mit der Balancirvorrichtung dienen dazu, den Druck, mit dem der Krystall auf der Schleifplatte aufliegt, je nach der Beschaffenheit des Präparates zu modificiren.

Zur Bequemlichkeit ist aussen nahe an dem Sttitzpunkte eines der Hebel eine justirbare Schraube o angebracht, die wie eine elektrische Klemmschraube - doch ohne seitliche Löcher - aussieht; der Cylinder, in dem die Schraube sich dreht, steht auf dem Theilkreise gerade unter dem längeren Hebelarme, so dass dieser durch den Schraubenkopf in beliebiger Höhe unterstutzt werden kann; der Hebel kann sich so in der Richtung, in der das Gegengewicht aufwärts geht, bewegen, während Bewegung in entgegengesetzter Richtung nicht möglich ist. An dem anderen Hebelarme ist ein Vorsprung $p$ fest angebracht, welcher unbequeme Senkung dieses Hebels verhindert. Die Leichtigkeit, mit welcher sich die Axe $i$ auf und ab bewegt, kann durch einen anderen gespaltenen Ring $q$ modificirt werden, welcher eine von dem Theilkreise ausgehende ebenfalls gespaltene Flansche umspannt, in der die Axe gleitet. Um ein sofortiges Auseinanderweichen des Ringes beim Lösen der Schraube zu bewirken, ist zwischen den beiden Ansätzen des Ringes, durch welche die Schraube geht, eine starke Feder angebracht.

Die Bronzeaxe $i$ ist inwendig auf die aus Fig. 2 ersichtliche Art durchbohrt; die Bohrung ist ziemlich weit für die oberen $\frac{2}{3}$ der Länge, enger in dem unteren Theile, und gestattet einer stählernen Kernaxe $r$ ohne seitliches Spielen auf und ab zu gleiten; unabhängige Bewegung dieser letzteren ist wieder durch eine Kerbung und entsprechenden Metallvorsprung wie bei der Axe $i$ verhindert. Diese Stahlaxe $r$ trägt an ihrem unteren Ende Krystallhalter und Justirvorrichtungen und endigt oben in einer grob geschnittenen Schraube. Mit einem an dem Schraubengehülse $t$ befestigten Schraubenkopfe $s$, welch ersteres durch die die Bohrung der Bronzeaxe $i$ verschliessende Kappe $u$ durchgeht, kann die Stahlaxe und der Krystall gehoben oder gesenkt werden, um letzteren von der Schleifplatte zu entfernen oder ihr zu nähern. Das oben herausragende Ende der Schraube ist durch eine Kappe $v$ 
geschützl, welche auf den Schraubenkopf $s$ aufgeschraubt wird. Auf dieser Kappe endlich kann noch ein becherförmiges Gefäss befestigt werden, das Fig. 1 zeigt, und das zur Aufnahme von Schrotkörnern oder Gewichten dient, wenn man den Druck des Krystalles auf die Schleifplatte vermehren will.

Der Centrir- und Justirapparat am unteren Ende der Stahlaxe $r$ besteht aus je zwei rechtwinklig zu einander wirkenden Centrir- und Justirvorricbtungen; die letzteren sind nach der zuerst von v. Lang angewendeten Methode eingerichtet, und bestehen aus Tangentialschrauben, die auf Kreismetallsectoren wirken. Diese Vorrichtungen mussen stärker construirt sein, als es für gewöhnliche goniometrische Zwecke gebräuchlich ist. Zum Centriren ist eine Vorrichtung in Anwendung gebracht worden, welche eine Zeit lang von der Firma Troughton \& Simms zu Centrirzwecken benutzt worden ist, und welche von denselben an dem von Miers*) beschriebenen Goniometer mit verticalem Theilkreise angebracht worden ist. Dieses Arrangement ist stärker construirt und die Schrauben lockern sich nicht so leicht, wie bei der gebräuchlichen rechtwinkligen Form. Das Centriren wird bewerkstelligt durch die gegenseitige Bewegung zweier Scheiben $w$ und $x$ um einander, und dieser beiden um eine dritte $y$. Die dritte Scheibe $y$ ist durch die Brucke $z$ fest mit dem unteren Ende der Stahlaxe $r$ verbunden. Die zweite Scheibe $x$ ist in der Năhe der Peripherie durch einen Zapfen mit $y$ verbunden, und die Bewegung von $x$ um $y$ wird bestimmt durch einen Bolzen, welcher in $x$ eingeschraubl ist und durch eine gekrummte Fuhrung durchgeht, die concentrisch zum Zapfen aus $y$ ausgeschnitten ist; fester Contact zwischen den beiden Scheiben wird erhalten durch ein federndes Stossblech, welches zwischen dem breiten Schraubenkopf des Bolzens und dem Tisch $y$ eingeklemmt ist. Die Rotation von $x$ um den Zapfen wird hervorgerufen durch Drehen der oberen Traversirschraube, welche diametral verläuft, senkrecht zu jener Verbindung des Zapfens und Bolzens steht und unter der Brücke z durchgeht. Das Ende der Schraube druckt gegen einen kurzen verticalen Stiel, der an der Scheibe $x$ befestigt ist und durch das centrale Loch in $y$ hindurchgeht, und dieser Stiel wird während des Zurückdrehens gegen das Ende der Schraube durch eine federnde Vorrichtung $w^{\prime}$ angepresst, welche der an der Feinstellvorrichtung des Theilkreises angebrachten gleicht. Die unterste Scheibe $w$ kann um die mittlere $x$ mil Hülfe einer ganz ähnlichen Vorrichtung durch Drehen der Schraube $x^{\prime}$ zum Rotiren gebracht werden; letztere steht rechtwinklig zur oberen Schraube.

Die Justirvorrichtungen $y^{\prime}$ und $z^{\prime}$ sind so eingerichtet, wie man sie gewöhnlich an den genaueren Goniometern sieht; Schrauben obne Ende, die an senkrecht zu einander angebrachten Kreissegmenten angreifen. In-

*) Mineralogical Magazine, März 1891, p. 214. 
dessen sind sie stärker als gewöhnlich gebaut und es ist besonder's darauf $\mathrm{zu}$ achten, dass die beiden Bewegungen sich möglichst genau unter einem Winkel von $90^{\circ}$ kreuzen. Eine neue Vorrichtung ist angebracht, die bezweckt, eine gewünschte Richtung im Krystalle zur Schleifplatte ganz genau parallel zu machen. Auf der einen Backe der Fubrung jedes Segmentes ist eine ebenso gebogene silberne Platte befestigt, an der eine Gradtheilung eingravirt ist. Das bewegliche Segment selbst trägt in der Mitle des Bogens auf einem Silberplättchen eine Marke; Theilung und Marke sind so fein, dass man mit Hülfe der Lupe $10^{\prime}$ genau abschăzen kann. Die Theilung der Scala geht von der Mitte aus und erstreckt sich nach jeder Seite bis zu $35^{0}$. Wenn die Segmente in der normalen Stellung sind, d. h. ihre Enden mit den Enden der Fuhrungen zusammenfallen, so steht die Marke auf $0^{0}$ ein; die Grösse der Bewegung beim Drehen der Juslirschrauben kann auf den beschriebenen Theilkreisen unmittelbar abgelesen werden.

Ausser diesem Paar von Justirbewegungen, deren Ebenen einen rechten Winkel mit einander bilden, ist ein zweites Paar vorhanden, bei dem die Ebenen der Kreisbewegung in irgend einen beliebigen Winkel zu einander gebracht werden können. Diese verstellbare in Fig. 3 dargestellte Justirvorrichtung ist nutzlich in gewissen selteneren Fällen monosymmetrischer Krystalle, in denen keine Flächen entwickelt sind, welche ein schnelles Justiren der gewunschten Elasticitätsaxe durch Kreisbewegung in rechtwinklig zu einander stehenden Ebenen gestatten; auch kann sie mit Vortheil bei asymmetrischen Krystallen verwendet werden. Es wird dieselbe Centrirvorrichtung gebraucht; man braucht bloss den rechtwinkligen Justirapparat zu entfernen, indem man die vier Schrauben herausnimmt, die seine Träger an der untersten der drei Centrirscheiben festhalten, und ihn durch den verstellbaren Apparat zu ersetzen. Die untere Kreisbewegung lässt sich in horizontaler Ebene gegen die obere drehen, und der Belrag dieser Drehung kann an einem Theilkreise abgelesen werden, der an dem oberen Segment angebracht ist; an dem unteren befindet sich an der Fubrung ein mit vier je $90^{\circ}$ von einander entfernten Marken versehener Kreis. Um Raum zum Anbringen dieser beiden Scheiben zu gewinnen, ist das obere Segment etwas grösser als bei den gewöhnlichen Justirapparaten gemacht. Der Theilkreis ist, wie die getheilten Bögen der Justirsegmente, in Grade eingetheilı; $10^{\prime}$ können leicht geschätzt werden. Wenn irgend eine der Marken mit dem Nullpunkte des Theilkreises correspondirt, so laufen die Justirbewegungen entweder parallel oder bilden einen Winkel von $90^{\circ}$; sind letztere gegen einander geneigt, so giebt eine Ablesung direct den Betrag dieser Neigung an. Die untere Vorrichtung kann in beliebiger Stellung an die obere mit einer Klemmschraube festgeklemmt werden; diese Schraube verbindet die markirte Scheibe mit dem Theilkreise und kann von beiden Seiten mit einem Schraubenschlussel angezogen werden. 
Der Krystall wird mit Hülfe eines leicht schmelzbaren, aber harten und schnell erstarrenden Wachses direct an einen kleinen Halter gekittet; derselbe besteht aus einem unten mit tiefen einander kreuzenden Rinnen

Fig. 3.

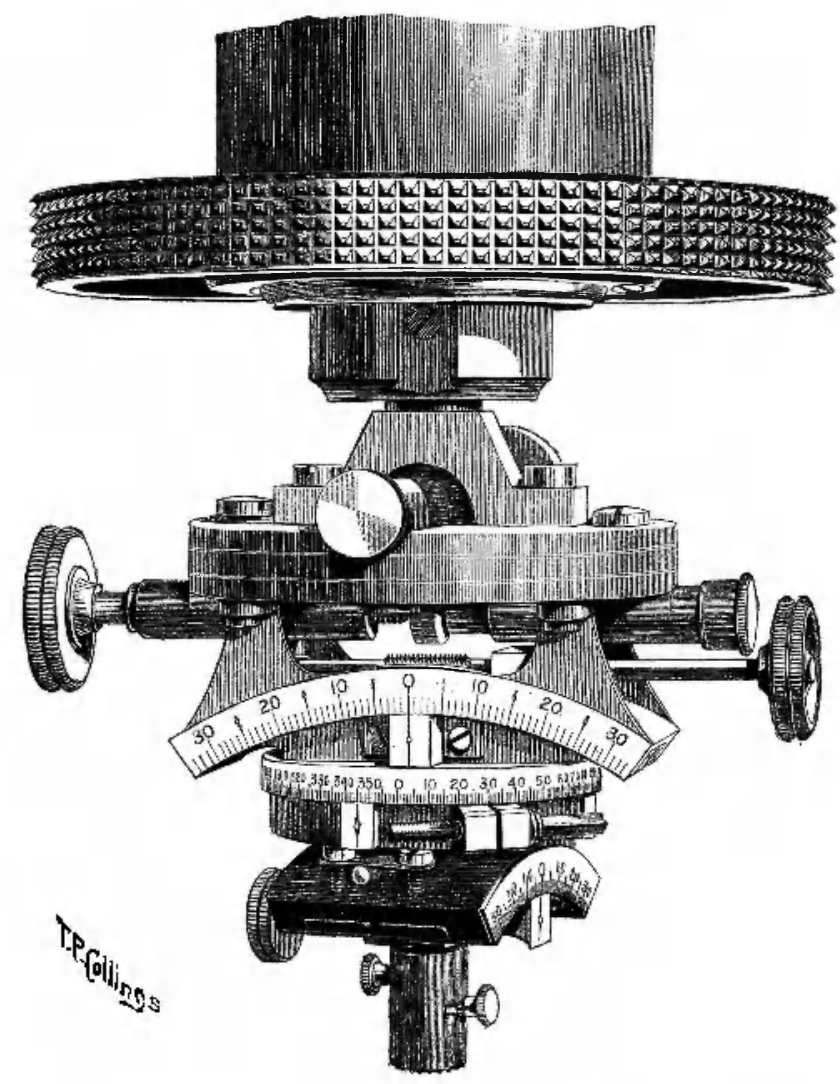

versehenen Messingtisch, um das Gleiten des Wachses zu vermeiden; ein in der Mitte befestigter kurzer Stahlstab passt in ein Loch unten im Justirapparate; derselbe hat eine Rinne, der eine Schiene in dem Loche entspricht, um das Rotiren zu vermeiden. Zwei solche Halter sind dem Instrument beigefügt, einer von $1 \mathrm{~cm}$ und der andere $i \frac{\tilde{i}}{i} \mathrm{~cm}$ im Durchmesser. Ausserdem sind noch zwei specielle Halter vorgesehen, von denen der eine den Krystall bis zu einem gewissen Grade zu drehen gestattet, um irgend eine Fläche desselben parallel zu einer den Tangentenschrauben justiren zu können, und der andere, von complicirterer Construction, dient dazu, eine zweite Fläche parallel zu einer schon angeschliffenen zu schleifen. Diese werden bei einer späteren Gelegenheit beschrieben werden.

Fernrohr und Collimator sind 2uf starken Stativen angebracht, welche 
auf einer kreisförmigen Führung gleiten, deren Krümmungsmittelpunkt in der Verticalaxe des Instrumentes liegt. Um eine möglichst freie Bewegung von Fernrohr und Collimator zu ermöglichen, sollen zum Tragen des Theilkreises und der Axen drei und nicht vier Säulen verwendet werden. Die unten gleitenden Flächen der das Fernrohr und den Collimator tragenden Stalive werden durch leicht gekrümmte Federn gegen die Fürung gepresst; diese Federn befinden sich innen zwischen der Ecke der Fuhrung und der Nuth der Gleitflächen. Fernrohr und Collimator können in derselben Horizontalebene justirt werden, welche senkrecht zur Axe des Instrumentes steht; gegen diese können die optischen Röhren genau gerichtet werden. Der äussere Haupttubus eines jeden trägt zu diesem Zwecke einen Ring, der an den stärkeren Ring des Trägers mit zwei Justirschrauben an dem äusseren Ende des Verticaldurchmessers angeschraubt ist; dadurch kann die Altitude schwach verändert werden, wäbrend der Azimuth durch eine dritte Justirschraube auf einer Seite, $90^{\circ}$ von den anderen entfernt, justirt werden kann. Fernrohr und Collimator gleiten in dem äusseren Tubus, so dass sie dem Krystall genähert oder von ihm entfernt werden können; sie können in irgend einer gewünschten Stellung mit Hülfe eines Spaltringes am äusseren Tubus festgeklemmt werden. Das Ocular des Fernrohres ist heweglich, um letzteres genau auf paralleles Licht einstellen zu können; das Fadenkreuz, in einem kurzen Rohre befindlich, ist mit feinen Stellschrauben mit dem Oculare verbunden. Die Stellung des Oculares kann, nachdem man dasselbe in die richtige Lage gebracht hat, mittelst eines Spaltringes fixirt werden, welch' letzterer einen Keil trägt, der in einen entsprechenden Schlitz am Objectivrobre hineinpasst. Hinten am Objectiv befindet sich, wie an den Fuess'schen Goniometern, eine bewegliche Linse, die vorgeschoben werden kann und dadurch das Fernrohr in ein schwach vergrösserndes Mik roskop verwandelt, um den Krystall betrachten zu können. Das Collimatorrohr trägt einen Websky'schen Spalt und kann ebenfalls verschoben und in beliebiger gewünschter Stellung mittelst eines Spaltringes fixirt werden. Fernrohr und Collimator können in irgend einen Winkel zu einander eingestellt und an ihrer Führung mit einer Schraube festgeklemmt werden.

Die Schleifplatte besteht aus einer runden Scheibe von mattgeschliffenem Glase und ist auf einer starken Trägerplatte von Messing mit aussen umgebogenen Kanten montirt. Die feingeschliffene Oberfläche des Glases muss möglichst genau eben sein. Die Trägerplatte ist auf eine Rolle aufgeschraubt, die fest an der starken Rotationsaxe angebracht ist; die letztere ragt ein wenig uber die obere Fläche der Rolle hinaus und passt genau in ein centrales Loch, das in die untere Seite der Messingträgerplatte gebohrt ist, um letztere concentrisch zu erhalten; unten läuft sie in einer genau cylindrisch gebohrten Stutze. Die Rotationsaxe muss naturlich zur 
Schleifplatte und zur Rolle sehr genau senkrecht stehen. Eine besondere Vorrichtung an dem cylindrischen Träger erlaubt die Schleifplatte genau senkrecht zur Drehungsaxe zu justiren, Vom oberen Theile jener Träger gehen drei Füsse aus, die in starken, direct auf der metallenen Basis ruhenden Nivellirschrauben endigen. Der Träger selbst geht unten durch ein ziemlich weites Loch in dem Tische, der seinerseits elwas höher ist als der zur Aufnahme eines Glassturzes beslimmle Holztisch; etwas unterhalb des Metalltisches endigt der Träger in einem breiten Kopf, und zwischen diesem und der unteren Fläche des Tisches ist eine starke Spiralfeder so angebracht, dass die drei Nivellirschrauben fest an den Melalltisch gepresst werden. Die Axe der Schleifplatte wird am Aufsteigen in der cylindrischen Bohrung des Trägers durch eine passende Flansche verhindert, und sowohl das breite obere Ende des Trägers als auch der Beschlag der Rolle, welcher darauf läuft, sind ganz eben gearbeitet. Durch ein seillich angebrachtes Röhrchen kann Schmieröl in die Bohrung des Trägers eingeführt werden. So läuft die Schleifplatte obne das geringste Stossen und mit einem Minimum von Reibung. Mittelst der Rolle rechts in Fig. 1 wird die Platte in Rolation versetzt; der Durchmesser dieser Rolle ist etwa derselbe wie der der Schleifplatte. Diese Rolle ist auf einem Träger in einer stabilen festen Säule montirt und mit einer passenden Handhabe zum Drehen versehen. Um den Druck auf beide Seiten der Axe der Schleifplatte möglichst gleichmässig zu machen, ist eine dritte Rolle von derselben Grösse wie diejenige unterhalb der letzteren angebracht; dieselbe ist ähnlich montirt, wie die grosse Triebrolle, doch ohne Handhabe. Die Transmissionsschnur von starkem Katzendarm ist auf beiden Seiten der mittleren Rolle gekreuzt; der Träger der dritten Rolle gleitet in einer kurzen Führung im Metalltische und kann mit einer starken Schraube unterbalb des Tisches bewegt werden, um die Schnur nachzulassen oder fester anzupressen. Das vortheilbafteste Grössenverhältniss der Rollen ist derart, dass auf eine Umdrehung der Triebrolle zwei der Schleifplatte kommen. Zu schnell darf man nicht drehen, denn die dadurch bewirkte unvermeidliche Erhilzung beim Schleifen stört immer, indem das Wachs weich wird und der Krystall seine Stellung ändert.

Man bedient sich vortheilhaft zweier Schleifplatten; die eine ist, wie oben beschrieben, fest angebracht und kann nicht entfernt werden; sie ist sehr fein angeschliffen, dass sie durchscheinend ist, und dient ausschliesslich zum Poliren der mit der anderen Platte angeschliffenen Flächen; die zweite Platte besleht aus dickem Glase; die Flächen sind eben und genau parallel geschliffen; die obere Fläche ist viel gröber matt geschliffen als die Oberfläche der festen Platte, etwa wie die Projectionsplatten an photographischen Apparaten. Diese Platte ist in einem starken Messingrahmen montirt, der drei Vorsprunge trägt, welche mit drei Vorsprungen in der Metallplatte correspondiren, auf die die Polirplatte aufgekiltet ist. Mit Schrauben, welche 
durch diese Vorsprünge durchgehen, kann die zweite Platte leicht uber der ersten befestigt und wieder entfernt werden; Schraubenmultern unterhalb der Metallplatte dienen zum Anziehen.

Nach längerem Gebrauche wird die gröbere Glasplatte matt werden und nicht mehr so schnell schleifen wie im Anfange. Man giebt ihr dann den fruheren Grad von Rauhigkeit wieder vermittelst einer kleinen mit einer Handhabe versehenen Platte (vorn in Fig. 1); diese ist eben geschliffen und wird mittelst eines passenden Bindemittels mit einer ganz dunnen Schicht feinsten Schmirgels uberzogen. Die Schleifplatte feuchtet man mit Terpentinöl an und bewegt die geschmirgelte Fläche parallel dem Durchmesser über die matten Stellen der Glasplatte bin; dies ist dem concentrischen Anschleifen vorzuziehen, weil dann keine Streifung auf der angeschliffenen Fläche am Krystall entsteht.

Das Schleifen nimmt man wegen der öfter zu wiederbolenden goniometrischen Messungen am besten in einem Dunkelraume vor; als Beleuchtungslampe ist ein Auer'sches Glühlicht zu empfehlen, das mit einer Vorrichtung ähnlich der an den Flürscheim'schen Brennern benutzten versehen ist. Als Goniometerlampe zur Beleuchtung des Spaltes bat sich als sehr praktisch die in der folgenden Abhandlung Figur 7 abgebildete Beleuchtungsvorrichtung, ebenfalls mil Auer'schem Glühlicht versehen, erwiesen.

\section{Justirung des Instrumentes.}

Man wird bemerkt bahen, dass jeder Theil des Instrumentes mit eigenen Justirvorrichtungen versehen ist, was die Handlichkeit und Genauigkeit beim Arbeiten wesentlich erhöht.

Das Fernrohr muss natürlich genau senkrecht zu der verticalen Rotationsaxe justirt werden; es geschieht dies in der gewöhnlichen Weise mit Hulfe der beiden Justirschrauben, die an dem Klemmringe angebracht sind; dieselben werden angezogen, bis die von einer planparallelen, auf beiden Seiten versilberten Glasplatte, die an dem Krystallbalter befestigt ist, reflectirten Fadenkreuzbilder genau mit dem im Fernrobre befindlichen Fadenkreuze zusammenfallen. Das Fernrohr muss dann noch für den Azimuth justirt werden; d. h. seine Axe muss genau auf die Rotationsaxe gerichtet werden, so dass sie dieselbe schneidet. Um dieses zu bewerkstelligen, wird der Krystallhalter durch ein kurzes, zugespitztes Stäbchen ersetzt (siehe Fig. 1, links auf der Basisplatte). Dasselbe wird vermittelst der Centrirschrauben centrirt, so dass die Spitze, wenn man sie durch das mit einer beweglichen Linse in ein Mikroskop verwandelte Fernrohr betrachtet, mit der Rotationsaxe zusammenfällt. Die seitliche Justirschraube des Teleskopringes wird dann so gedreht, dass die Spitze die Mitte des Gesichtsfeldes einnimmt. 
Die Justirung des Collimators wird dann leicht durch Drehen der Schraube seines Klemmringes bewerkstelligt, bis das Bild des erleuchteten Spaltes, direct durch das auf denselben gerichtete Fernrohr betrachtet, klar und vollkommen aufrecht erscheint und von dem horizontalen Theile des Fadenkreuzes genau halbirt wird.

Das Justiren der Schleifplatte parallel zu der Ebene von Fernrohr- und Collimatoraxe, aber senkrecht zur Rotationsaxe, wird folgendermassen ausgefubrt. Man uberzeugt sich zunächst, dass die Ebene von Fernrobr- und Collimatoraxe für alle Stellungen in der kreisförmigen Lagerfubrung senkrecht zur Rotationsaxe bleibt. Das Fernrohr wird dann ganz rechts in der Fuhrung festgeklemmt, so dass es fast die Säule berührt, uhd der Collimator wird zum nächsten Ende seiner Fubrung gebracht, so dass der Winkel zwischen beiden ca. $120^{\circ}$ beträgt. Auf die Schleifplatte legt man sodann einen Glaswúrfel von ca. $2 \mathrm{~cm}$ Seitenlänge, ein Glasprisma von genau $90^{\circ}$ brechendem Winkel oder einen passenden Krystall, der zwei einander genau unter $90^{\circ}$ schneidende Flächen aufweist, mit der einen dieser Flächen auf und zwar in solcher Lage, dass man mit dem Fernrohr das an der zweiten, senkrecht stehenden Fläche des Prismas reflectirte Collimatorbild beobachten kann. Dasselbe muss, wenn die Platte die richtige Stellung hat, vom horizontalen Theile des Fadenkreuzes genau halbirt werden. Man uberzeugt sich, dass dies für die ganze Oberfläche del Schleifplatte der Fall ist, indem man das Fernrohr und den Collimator an das andere Ende ibrer Führung. bringt, die Lage des Prismas um $90^{\circ}$ entsprechend ändert, und wieder beobachtet. Eventuell muss mittelst der Nivellirschrauben der Platte mit einem passend geformten Schraubenzieher justirt werden.

\section{Das Schleifen der ersten oberfläche einer Platte.}

Es sollen im Folgenden vier typische, beim Gebrauch des Instrumentes hauptsächlich in Frage kommende Fälle beschrieben und an Beispielen zweiaxiger Krystalle erläutert werden.

1. Der einfachste Fall ist der eines dem rhombischen Systeme angehörenden Krystalles, der eine woblausgebildete, die beiden Pinakoide enthaltende Zone zeigt, oder auch nur Prismen und Domen hat. Die Axe dieser Zone möge die Bisectrix sein, zu welcher senkrecht man eine Platte schleifen will; Flăchen parallel zu der fraglichen Ebene seien nicht vorbanden oder schlecht ausgebildet. Der Krystall wird mit leicht schmelzbarem, schnell erstarrendem Wachs auf dem Krystallhalter befestigt; er muss gut in das Wachs eingebettet sein, das man, während es noch warm ist, fest an den Krystall und in die Vertiefung des Halters hineindruckt; geschieht dies nicht, so zerbricht der Krystall leicht beim Schleifen. Beim Aufsetzen muss man darauf achten, dass die Axe der erwähnten Zone approximativ parallel der Goniometeraxe justirt ist. Man centrirt und justirt nun endgultig, gerade so 
als ob man eine goniometrische Messung vornebmen wollte; eventuell uberzeugt man sich durch Messen einiger Winkel, dass man die richtige Zone eingestellt hat.

Während dieser Operationen wird jede senkrechte Bewegung des Krystalles, um ihn auf die Höhe der Fernrohraxe zu bringen, mittelst der inneren Stahlaxe $r$ ausgefubrt, während die Bronzeaxe $i$ in der Weise fixirt bleibt, dass der Arm $p$ des Hebels, der sie trägt, auf der Theilkreisplatte aufliegt. Durch Dreben der Schraube o muss dem anderen Hebel eine nahezu horizontale Lage gegeben werden. Man wird nun bemerken, dass, während das Ansatzstúck des vorderen Hebels auf dem Theilkreise rubt, dessen kurzer, gekrümmter Arm allein die Axe trägt, während die stumpfe Schneide von dem kurzen Arme des horizontalen Hebels etwa $\frac{1}{2} \mathrm{~cm}$ unterhalb des an der Axe befestigten Ringes sich befindet. Wenn man nun den mit dem Ansatzstuck versehenen Hebel vorsichtig hebt, so fällt die Bronzeaxe, bis der Ring auch auf die Schneide des horizontalen Hebels aufstösst, und bei einer weiteren Abwärtsbewegung der Axe wird ihr ganzes Gewicht durch die beiden Hebel balancirt. Alsdann kann man das Gewicht oberhalb des Krystalles je nach der Festigkeit desselben beliebig erhöhen. Die Schleifplatte wird nun auf der Polirplatte befestigt und einige Tropfen eines dunnen Oels auf dieselbe gebracht. Das Oel vertheilt man am besten uber den Theil der Oberfläche der Platte, wo das Scbleifen stattfindet, mit einem kleinen Pinsel, der an einem beweglichen Gestell befestigt ist; der Pinsel dient zu gleicher Zeit dazu, die Platte und den Krystall zu säubern.

Die innere Axe wird sodann beruntergeschraubt, bis der Krystall nur noch wenig von der Schleifplatte entfernt ist, während man mit der linken Hand den Hebel hinunterdruckt, so dass das Ansatzstuck auf dem Theilkreise verbleibt. Der Hebel wird dann sehr vorsichlig gehoben, bis der Krystall die Schleifplatte gerade berührt, die dann in zuerst sehr langsame Rotation versetzt wird. Wenn der Krystall nicht äusserst zerbrechlich ist, kann der horizontale zweite Hebel ausser Action bleiben, bis das Schleifen beinahe vollendet ist, denn der Ring der Axe wird noch einige Millimeter böher sein als die Schneide des zweiten Hebels. Der Maximaldruck auf den Krystall ist dann ungefähr das halbe Gewicht der Axe; die meisten künstlichen Krystalle zerbrechen unter diesem Drucke nicht. Uebrigens kann das Gewicht, mit dem der Krystall auf die Schleifplatte gedrickt wird, sehr gut regulirt werden, indem man das Gregengewicht des Hebels mit Daumen und Zeigefinger der linken Hand fasst und dasselbe hebt oder einen leisen Druck auf dasselbe ausübt. Wenn der Krystall so weit abgeschliffen ist, dass er mit der Schleifplatte nicht mehr in Contact ist, so stellt man diesen ebenfalls durch vorsichtiges Heben des längeren Hebelarmes wieder her; selbst bei sehr zerbrechlichen Krystallen kann dies öfter geschehen, ohne die Krystallplatte zu verletzen. 
Bei ziemlich harten Krystallen, wie Kaliumsulfat, kann man die Wirkung des Gregengewichtes alle paar Secunden ganz aufheben, indem man den Hebel ausser Aclion setzt, ohne befürchten zu müssen, ein gutes Exemplar zu zerbrechen; doch muss die Drehung der Schleifplatte gleichmässig erfolgen und die Zahl der Umdrehungen pro Secunde darf höchstens zwei sein. Bei noch härteren Krystallen, solchen die eben weicher sind als Glas, kann das Gefäss am oberen Ende der Axe mit Schrotkörnern oder dergleichen beschwert werden, aber das Schleifen muss langsam erfolgen und muss aufmerksam iberwacht werden. Wenn im Gegentheil der Krystall sebr weich oder spröde ist, müssen beide Hebel in Action treten, beim horizontalen muss man die Stutzschraube niedriger stellen und mittelst des anderen durch Manipuliren des Ansatzes den Druck sorgfältigst reguliren. Bei vollkommener Spaltbarkeit tritt Zersplittern naturlich am leichtesten ein, wenn das Schleifen senkrecht zur Spaltungsrichtung erfolgt.

In allen Fällen ist es gut, bei Beendigung des Schleifens beide Hebel in Action treten zu lassen, weil dann die angeschliffene Fläche glatter wird und man nachher beim Poliren Zeit erspart. Von Zeil zu Zeit nimmt man den Krystallhalter heraus, um nachzusehen, ob der Krystall weib genug abgeschliffen ist; man kann dies ausfuhren, ohne fürchten zu müssen, die Justirung zu verderben, wenn die Einkerbung im Ansatz des Halters gut in die Führung hineinpasst. Wenn man weit genug abgeschliffen hat, muss man vor Entfernung der Schleifplatte immer noch einmal sich uberzeugen, ob der Krystall noch gut justirt ist, indem man ihn mit einem seidenen Tuche von Oel u. s. f. reinigt und mit der Goniometervorrichtung die Bilder der Flächen der eingestellten Zone revidirt. Meist wird die Justirung tadellos geblieben sein; solle durch zu schnelles Schleifen das Wachs sich elwas erwärmt haben und weich'geworden sein, so verschiebt sich zuweilen der Krystall etwas; in diesem Falle muss man neu justiren und wieder eine kurze Zeit schleifen, bis der Fehler behoben ist. Dann entfernt man die Schleifplatte und polirt mittelst der nicht entfernbaren unteren Polirscheibe, die man mit Oel benetzt; der Druck, den sie auf den Krystall ausubt, ist natürlich mit dem Hebel möglichst zu vermindern. Man benöthigt meist zum Schleifen 15 und zum Poliren 5 Minuten und erhält dann eine glasartige Oberfläche; dies ist wichtig, weil man dann die Axenwinkel messen kann, ohne Deckgläschen aufkitten zu müssen.

Auch nach dem Poliren sollte man die Justirung eigentlich immer noch einmal revidiren. Bemerkt muss werden, dass man die Platten niemals beim Schleifen mit einem Lösungsmittel der betreffenden Substanz benetzen sollte, da so die Krystallflächen verätzt werden und ein Revidiren der Justirung illusorisch gemacht wird.

2. Als zweiter typischer Fall mag ein monosymmetrischer Krystall betrachtet werden, an dem die Symmetrieebene vorherrscht; eine Unter- 
suchung im convergenten Lichte und Bestimmung der Auslöschungsrichtung mögen ergeben haben, dass die Mittellinie, senkrecht zu der wir eine Fläche anzuschleifen wünschen, einen bestimmten Winkel, kleiner als $45^{\circ}$, mit der Kante der Symmetrieebene und einem Prisma, dem Orthopinakoid, einem Doma oder der Basis bildet. Vier Operationen sind nöthig, um einen solchen Krystall so zu justiren, dass die bekannte Richtung der Mittellinie zur Schleifplatte senkrecht steht. Der Krystall muss zunächst so auf dem Halter festgegossen werden, dass die Flächenzone, welche der soeben erwähnten Kante parallel ist, ungefähr senkrecht zur Schleifplatte steht. In zweiter Linie muss die Symmetrieebene der oberen Tangentenschraube des Justirapparates genau parallel sein. Alsdann muss die ganze Zone genau senkrecht zur Schleifplatte justirt werden und es bleibt als vierte Operation nur übrig, die Tangentenschraube zu drehen und das Segment um die gewünschte Anzahl von Graden zu bewegen, bis die Mittellinie zur Schleifplatte senkrecht steht.

Fưr alle Fälle, in denen eine Krystallfläche zu einer Tangentenschraube parallel justirt werden soll, ist ein besonderer Krystallhalter vorgesehen, der, nachdem er mit dem Krystall eingefügt ist, um beinabe $90^{\circ}$ gedreht und in jeder beliebigen Stellung festgestellt werden kann. Die zwei Theile dieses Halters sieht man in Fig. 1, links von dem gewöhnlichen grossen Halter; in Fig. 3 ist er am Apparat eingesetzt. Er besteht aus einem gekerbten Stahlstabe, ähnlich wie die anderen Halter, und trägt unten einen kleinen compacten Stablcylinder. Der letztere passt genau in einen äusseren, hohlen, unten geschlossenen Cylinder; die Aussenseite der Endigung ist kreuzweise gekerbt, wie die Platten der anderen Halter, um das Wachs fester zu halten, in das der Krystall eingegossen wird. Dieser hohle Cylinder ist von zwei horizontalen Schlitzen von etwas mehr als $90^{\circ}$ Ausdehnung durchbrochen, und zwar auf verschiedener Höhe an entgegengesetzten Seiten des Cylinders. Der äussere wird in seiner Stelle um den inneren Cylinder gebalten durch zwei kleine Schrauben, welche durch die Schlitze auf den compacten Gylinder auftreffen; die Richtung der Scbrauben ist ungefähr parallel der oberen Tangenten-Justirschraube, wenn der Halter eingefügt ist. Nach der Justirung des Krystalles können diese Schrauben dazu dienen, den äusseren Cylinder an den compacten Kerncylinder festzuklemmen.

Um die vier oben näher bezeichneten Operationen auszufuhren, wird der Krystall auf bekannte Weise am Ende des hohlen Cylinders mit Wachs festgekittet, so, dass die Axe der zu justirenden Flächenzone der Axe des Cylinders nahezu parallel ist, und die Symmetrieebene nabezu parallel steht der Richtung der in der Mitte der Schlitze angebrachten Klemmschrauben. Das Wachs, das man benutzt, wird so schnell fest, dass nur eine grobe Annäherung an die richtige Stellung möglich ist, welche indessen völlig 
genügt. Am besten nimmt man die Operation mit eingeschobenem inneren Cylinder vor. Um eine Fläche genau parallel zu einer der 'Tangentenschrauben zu justiren, wird ein kleines Stück eines Deckgläschens auf der Fläche des niedersten Theiles des unteren Segmentes aufgekittet, unmittelbar oberhalb der Stelle, die der Krystallhalter einnimmt und parallel zu der Ebene, in der sich das Segment bewegt. Bevor man den cylindrischen Krystallhalter befestigt, wird die Axe hinuntergeschraubt, bis die Glasplatte sich in der Höbe der Axe des Fernrohres und Collimators befindet, das von derselben reflectirte Spaltbild wird genau eingestellt und abgelesen. Nun wird der cylindrische Krystallträger befestigt, der Theilkreis auf die obige Ablesung eingestellt, wenn die betreffende Fläche der unteren Tangentialschraube, um $90^{\circ}$ entfernt, wenn sie der oberen Tangentialschraube parallel sein soll, und der äussere Cylinder des Krystallhalters wird nun gedreht, bis das von der Krystallfäche reflectirte Spaltbild genau auf das . Fadenkreuz einsteht. Justiren kann man mit der zweiten, der Krystallfläche nicht parallelen Tangentialschraube. Dann wird der Cylinder in seiner Bohrung mit den zwei kleinen Klemmschrauben befestigt.

Hat man so die Symmetrieebene parallel zu der oberen Tangentialschraube justirt, so nimmt man mit eben dieser Schraube die Justirung der ubrigen Flächen der Zone senkrecht zur Schleifplatte vor. Endlich wird die Stellung des oberen Segmentes an dem daran angebrachten Theilkreise abgelesen und dessen Schraube um den gewünschten Bogen (der Auslöschungswinkel in Bezug auf die Axe der Zone) gedreht, und auf $10^{\prime}$ genau eingestellt, bis die Richtung der Bisectrix senkrecht zur Schleifplatte steht. Schleifen und Poliren wird dann genau wie oben angegeben ausgeführt.

3. Als nächster Fall möge ein rhombischer Krystall betrachtet werden, der ein Pinakoid, sonst nur prismatische, oder Pyramiderflächen zeigt. Es seien die Basis und die vier einer Form angehörigen Prismenflächen entwickelt, und man soll eine Fläche parallel zu einem der nicht vorhandenen Pinakoide schleifen. Dann justirt man die Basis parallel zu der oberen Tangentialschraube und die Zone, die gebildet ist durch die Basis und ein Paar der Prismenflächen senkrecht zur Schleifplatte, wie oben beschrieben. Kennt man den Prismenwinkel, so braucbt man die obere Justirschraube nur in der passenden Richtung um die Hälfte dieses Winkels zu drehen, um das gewünschte Pinakoid in parallele Lage zur Schleifplatte zu bringen. Ist der Prismenwinkel sehr spitz, so dass man um einen grossen Winkel drehen muss, so stellt man die obere Justirschraube nicht auf Null, sondern auf einen Winkel in der entgegengesetzten Richtung von derjenigen, in welcher die Drehung erfolgen muss, ein.

Ganz analog ist zu verfahren, wenn ein rhombischer Krystall uberhaupt 
keine Symmetrieebene zeigt; man justirt dann eine passende Fläche zu einer der Justirschrauben und dreht um den Winkel, den man zuvor berechnet hat, in der passenden Richtung.

4. Hat man einen monosymmetrischen Krystall, an dem die Symmetrieebene gar nicht, sondern nur prismatische Formen entwickelt sind, so wird die in Fig. 3 abgebildete specielle Form des Justirapparates gute Dienste leisten. Man schleift dann die Symmetrieebene an, indem man einfach ein Flächenpaar aus der Orthodomenzone senkrecht zur Axe des Instrumentes justirt. Hat man aber eine Fläche senkrecht zu einer optischen Elasticitätsaxe zu schleifen, die, der Symmetrieebene parallel, einen Winke] von weniger als $45^{0}$ mit der Verticalaxe des Krystalles bildet, so verfährt man nach Fall 2, wenn die Symmetrieebene entwickelt ist; wenn das Orthopinakoid ausgebildet ist, bietet der Fall ebenfalls keinerlei Schwierigkeit. Sind indessen nur prismatische Flächen in der Verticalzone vorhanden, so ist es einleuchtend, dass zwei zu einander senkrechte Drehbewegungen nicht ausreichen, um die Mittellinie in eine verticale Lage zu bringen, doch kann man die Symmetrieebene leicht in ihre richtige Lage bringen durch zwei gleiche Bewegungen in Ebenen, welche gleich geneigt sind gegen die Symmetrieebene. Der besondere Justirapparat, mittelst dessen die untere Kreisbewegung in irgend eine gewünschte Neigung zur Ebene der oberen gebracht werden kann (für gewöhnlich bilden beide einen Winkel von $90^{\circ}$ mit einander), setzt uns in den Stand, dies auszufubren. Es ist nur nothwendig, die beiden Justirbewegungen zwei Prismenflächen derselben Form parallel zu stellen, je eine auf jeder Seite der Symmetrieebene, und die Segmente mit Hülfe der Tangentialschraube um die berechnete Zahl von Graden zu drehen. Die Berechnung ist eine sehr einfache, da ja der Gesammtbetrag der gewúnschten Drehung in der Symmetrieebene (der Auslöschungswinkel) und die Neigung der beiden Kreisbewegungen gegen diese Fläche bekannt ist. Um die Bewegungen den beiden Prismenflächen parallel zu stellen, genügt es, die eine der unteren Tangentialschrauben in bekannter Art parallel zu stellen, und dann anfangend vom Nullpunkte des Theilkreises (beide Bewegungen parallel) die untere gegen die obere um die dem bekannten Prismenwinkel entsprechende Anzahl von Graden zu drehen, die auf dem kleinen horizontalen Theilkreise an der Justirvorrichtung abzulesen sind. $\mathrm{Zu}$ empfehlen ist, den Krystall schon von vornherein so richtig als möglich aufzukitten.

Man kann auch auf andere Weise noch bequemer zum Ziele gelangen. Eine der Prismenflächen wird der unteren Tangentialschraube parallel gemacht, hierauf das untere Segment um das obere um den Winkel zwiscben Prisma und Symmetrieebene so gedreht, dass die letztere der oberen Tangentialschraube parallel steht. Man justirt dann die Prismenzone genau parallel zur Rotationsaxe, und kann dann die betreffende Bisectrix obne 
weiteres durch Drehung des oberen Segments um die Auslöschungsrichtung in eine senkrechte Lage zur Schleifplatte bringen.

Schwieriger als die beschriebenen Fälle ist natürlich ein trikliner Krystall zu behandeln, und eine allgemeine Vorschrift für einen solchen Fall kann selbstredend nicht gegeben werden. Am besten verfertigt man sich eine exacte sphärische Projection des Krystalles, in die man die vorhandenen Flächen, die optischen Mittellinien und Axen einträgt; nach einiger Uebung kann man mit Hülle einer solchen Projection rein mechanisch mittelst der an dem Instrument angebrachten Justirvorrichtungen und Theilkreise die gewúnschte Richtung senkrecht zur Schleifebene bringen.

\section{Schleifen der zweiten Fläche parallel zur ersten.}

Ist eine Fläche fertig geschliflen, so kann man die zweite mit dem Eingangs erwähnten kleinen Apparate von Fuess parallel anschleifen; bequemer und genauer lässt sich dies jedoch mit meinem Apparate mit Hülfe eines besonders zu diesem Zwecke construirten Krystallhalters erreichen.

Zunächst muss man den Krystall mit der angeschliffenen und gut polirten Fläche auf ein kreisförmiges, planparalleles Glasscheibchen aufkitten, das etwa $\frac{5}{4} \mathrm{~cm}$ Durchmesser hat und wovon man am besten eine grössere Zahl aus dünnstem Objectträgerglase sich schneiden lässt.; Deckgläschen sind zu zerbrechlich. Als Kittmittel verwendet man harten Canadabalsam, den man, wenn eine Veränderung der Krystallsubstanz nicht zu befürchten ist, auf $60-70^{\circ}$ erhitzt; sonst muss man eine Benzollösung von Canadabalsam verwenden und warten, bis derselbe bei gewöbnlicher Temperatur hart geworden ist, was im Allgemeinen nicht länger als 12-24 Stunden dauert. Hat man den Krystall so auf die Glasplatte aufgekittet, so setzt man dieselbe in den Träger ein, der kurz beschrieben werden soll.

Er besteht aus zwei Theilen, die man in Fig. 1 vorn auf dem Tische etwas rechts sieht. Der obere Theil (rechts in der Figur) besteht aus einer dicken Messingscheibe, $2 \frac{1}{2} \mathrm{~cm}$ im Durchmesser, die eine Seite ist genau eben gearbeitet und auf der anderen ist in der Mitte ein gekerbter Stahlansalz wie bei den anderen Haltern genau senkrecht eingesetzt. Auf derjenigen Seite, auf welcher sich der Ansatz befindet, ist eine niedrige Kappe von weissem, hartem Metall mit drei Schrauben befestigt; sie uberdeckt die dicke Scheibe bis zur Hälfte und dehnt sich nach auswärts etwa $0,6 \mathrm{~mm}$ in eine der ebenen Oberfläche parallele Flansche aus. Diese Flansche ist an drei symmetrisch gelegenen Punkten durchbohrt. Der untere Theil, ganz von sehr hartem, weissem Metall gearbeitet, gleicht der Kappe in der Form, und die unbedeckte untere Hälfte der dicken Scheibe 
passt sehr genau hinein; die äussere Flansche ist von gleichem Durchmesser wie die der Kappe und trägt drei feste Schrauben, die durch die Löcher der oberen Kappe hindurchgehen. In der Mitte der unteren Kappe ist eine runde Vertiefung, in welche die Glasplättchen bineinpassen. Ein etwas kleineres concentrisches Loch ist hineingeschnitten; die Dicke der Kappe ist so gewählt, dass der kleine Ring, der bleibt, um die Glasplättchen zu tragen, etwa papierdick ist.

Will man den Träger benutzen, so fügt man die obere Hälfte unten in das Instrument ein, justirt die ebene Oberfläche der Messingscheibe parallel zur Schleifplatte, indem man hinter der letzleren einen weissen Schirm aufstellt und zwischen den beiden einander sehr genäherten Ebenen durchvisirt. Dann fügt man die untere Hälfte mit der Glasplatte und dem Krystalle ein, letzterer durch das Loch hervorragend, schraubt mit drei Schraubenmuttern die beiden Theile des Halters aneinander und schleift wie oben angegeben, bis die Krystallplatte die gewünschte Dicke hat, um die Interferenzfigur im convergenten Lichte gut zu zeigen. Wenn man die Platte gut aufgekittet hat, so werden die zwei Flächen schön parallel werden; natürlich kann man mit der Goniometervorrichtung meist an den vorhandenen Krystallflächen prúfen, ob der Krystall die richtige Stellung hat.

Die Dúnne der so erbaltenen Platien ist naturlich beschränkt durch die Dicke des Ringes, auf welchem die Glasplatle aufliegt; selten wird die Doppelbrechung so stark sein, dass man die Platte nicht genügend fein erhält. Sollte dies einmal vorkommen, so kann man die Glasplatte direct auf die ebene Fläche der Messingplatte aufkitten.

Man entfernt, wenn man mit Schleifen und Poliren fertig ist, die Krystallplatte vom Glase durch Auflösen des Balsams in Benzol und kittet sie auf ein schmales Glasplättchen oder besser bält sie in einer kleinen Pincette aus Platinblech fest, um den optischen Axenwinkel zu messen. Soll dies in $\alpha$-Bromnaphtalin geschehen, so kittet man am besten mit Marineleim (marine glue) auf, da Canadabalsam gelöst werden würde.

\section{Das Schleifen von Prismen.}

Diese Operation kann eben so schnell und einfach ausgeführt werden, wie das Schleifen von Platten. Meist wird man eine Prisma von etwa $60^{\circ}$ brechendem Winkel brauchen, dessen Ebenen gleich geneigt sind gegen eine Haupt-Elasticitäts@äche. Man justirt dann diese Ebene parallel zur Schleifplatte und die Kante des Prismas parallel zur unteren Tangentialschraube. Dann dreht man die obere Schraube um $30^{\circ}$, schleift eine Fläche an, entfernt den Krystall vom Halter, setzt ihn in umgekehrter Stellung wieder ein, justirt wie oben, dreht um $30^{\circ}$ und scbleift die zweite Prismenfläche an. Wenn der Krystall nicht sehr klein ist, so kann man auf beiden 
$4 \tilde{5}$ A. E. Tutton. Ueb. ein Instrument zum Schleifen von genau orient. Platten etc.

Seiten je ein Prisma anschleifen und Controllmessungen vornehmen, was manchmal empfehlenswerth ist. Auch ist es ganz leicht, ein anderes Prismenpaar, symmetrisch zu einer anderen optischen Elasticitätsebene anzuschleifen, so dass alle drei Brechungsexponenten an einem Krystall bestimmbar sind.

Das Bedecken der Prisnenflächen mit Deckgläschen hat sich durchweg als unnöthig erwiesen; die Spaltbilder, welche die angeschliffenen Flächen reflectiren, sind immer schon hell und ganz einheitlich.

Zum Schlusse mag bemerkt werden, dass zum Schleifen harter Körper, natürlich vorkommender Krystalle, das Instrument nicht gebraucht werden kann; der Verfasser wird in Kurzem einen für diesen Zweck construirten ähnlichen Apparat beschreiben.

Der Verfasser spricht dem »Research Fund Committee « der Chemical Society, welcher die Kosten für die Construction bewilligte, seinen Dank aus; das Instrument wurde construirt von Troughton und Simms in London. 